\title{
Ailleurs Plutôt QUe NUlle Part. L'image de la Pologne et des Polonais goyim dans les textes D'IMMIGRÉS JUIFS D'ORIGINE POLONAISE ET DE LEURS DESCENDANTS EN BELGIQUE FRANCOPHONE
}

\author{
Przemysław Szczur ${ }^{1}$ \\ https://orcid.org/0000-0001-9474-5887 \\ (Université Pédagogique de Cracovie) \\ e-mail: przemyslaw.szczur@up.krakow.pl
}

Keywords: Belgian literature in French, Belgium, immigrants, Poland, Poles, Polish Jews

\begin{abstract}
This paper analyses the image of Poland and gentile Poles in the texts of Jewish immigrants and their descendants in French-speaking Belgium. The centre of interest are its literary forms, as well as its historical roots. If the geographical and cultural Polish space appears alternatively as locus amoenus and locus horribilis, the inhabitants of the country are quite clearly represented as populus horribilis. Their antisemitism appears as one of their main features, although sometimes it is problematized. Jewish immigrants and their descendants distance themselves from Poland and gentile Poles. They mainly create a negative image, very different from the traditional image of Poland and Poles in Belgian literature.
\end{abstract}

\section{Introduction}

Au début de la pièce Ä̈da vaincue de René Kalisky, le souvenir de la Pologne fait l'objet d'un court échange entre l'héroïne principale et sa mère : «AÏDA. [...] Toi, tu n'es [...] partie qu'une fois dans ta vie. Mais tu n'as jamais regretté la Pologne, hein ? LA MÈRE. Oh, tu sais... c'est si loin. Je ne me la rappelle plus, la Pologne ${ }^{2}$ ». Ce dialogue pose la question de savoir ce qui reste du pays d'origine dans la mémoire de l'émigré(e) et y fournit une réponse sur le mode négatif. Si la mère d'Aïda semble frappée d'une curieuse amnésie en ce qui concerne la Pologne, il n'en est pas toujours de même pour les autres personnages et narrateurs d'œuvres d'immigrés juifs d'origine polonaise et de leurs descendants en Belgique francophone. La Pologne et les Polonais y sont parfois davantage présents. Je me pencherai, dans ce texte, sur les formes et sens de cette présence, dans un corpus essentiellement contemporain, comprenant surtout des œuvres des années 1990 et 2000, quand a eu lieu une sorte d' " explosion discursive » autour de la thématique migratoire dans les lettres belges francophones, mais également quelques ouvrages

1 L'auteur de cette étude bénéficie d'un financement octroyé par le Centre National de la Recherche Scientifique Polonais (National Science Centre, Poland, research project 2018/30/M/HS3/00153).

2 Kalisky 1990:11. 
plus anciens ${ }^{3}$. Il s'agit de textes appartenant à différents genres - roman, recueil de nouvelles, essai, scénario de film, pièce de théâtre, autobiographie, bande dessinée... - et que relient les origines juives polonaises de leurs auteur(e)s ainsi que la présence en leur sein de références à la Pologne et aux Polonais. Par leur date de publication, toutes ces œuvres appartiennent à l'époque post-Shoah. L'on sait, notamment grâce aux analyses de Havi Ben-Sasson ${ }^{4}$, que l'Holocauste a profondément modifié l'image des Polonais goyim ${ }^{5}$ parmi les Juifs polonais. Il me semble intéressant d'étendre l'examen de cette image aux textes de Juifs d'origine polonaise établis en Belgique, publiés après cette époque charnière, comme cela a déjà été fait pour ceux qui se sont fixés en Israël ${ }^{6}$. Cette recherche voudrait ainsi contribuer, à sa modeste mesure, à combler une lacune encore récemment constatée par Audrey Kichelewski qui rappelait l'inexistence d'une étude d'ensemble sur les représentations de la Pologne parmi les émigrants juifs polonais après $1945^{7}$. En essayant de scruter les particularités de la vision de leur pays d'origine chez ceux qui se sont installés en Belgique francophone et chez leurs descendants, je la confronterai à des travaux historiques, car ce qui m'intéresse, ce sont aussi les racines factuelles de l'image de la Pologne et des Polonais goyim créée par les immigré(e)s. Comme, à quelques exceptions près, il s'agit de textes peu connus, je les citerai assez abondamment.

\section{Des toponymes polonais à la présentation chronotopique de la Pologne}

La Pologne en tant qu'espace géographique est surtout évoquée dans les textes du corpus à travers des toponymes. Les lieux de naissance et de résidence des auteur(e) s ou de leurs ancêtres, ainsi que ceux de leurs personnages, sont mentionnés, situés plus

${ }^{3}$ Le corpus se compose, dans l'ordre chronologique, de : L'apprentissage inutile de David Scheinert (1948; roman), L'immigré Herschel Schaerbeeker raconte de Zalek Kalb Beller (1978; recueil de nouvelles), « Belgique ? Le pays le plus imaginaire du monde » de René Kalisky (1981 ; essai), Bruxelles-transit de Samy Szlingerbaum (1989; scénario de film), J'avais 16 ans à Auschwitz d'Henri Sonnenbluck (1990 ; autobiographie), Ä̈da vaincue de René Kalisky (1990 ; pièce de théâtre), Eva, Rachel, Yitzhak et les autres... De l'émigration polonaise à l'enfant caché d'Anna Stelkowicz (1997; pièce de théâtre), Une adolescence perdue dans la nuit des camps d'Henri Kichka (2005 ; autobiographie), Écris, papa, écris d'Elie Rozencwajg (2005; autobiographie), Plus que la vie de Charles Lerner (2007 ; autobiographie), Bubelè. L'enfant à l'ombre d'Adolphe Nysenholc (2007; roman), Témoignage de Sylvain Gutmacher (2010 ; roman autobiographique), Le livre de Joseph de Bernard Dan (2011; roman), Deuxième génération. Ce que je n'ai pas dit à mon père de Michel Kichka (2012 ; bande dessinée), Monsieur Optimiste d'Alain Berenboom (2013 ; autobiographie), La danseuse sur un nuage de Regina-Sylvia Cykiert (2013; autobiographie), Wanda. De la Sibérie à Anvers, le courage de la différence de Jerzy Hildebrand (2013; autobiographie) et Un monde sur mesure de Nathalie Skowronek (2017; autobiographie).

4 Ben-Sasson 2009.

5 J'adopte ici ce terme pour pouvoir renvoyer à tous les Polonais non juifs (catholiques, protestants, etc.) et éviter la distinction entre « Juifs » et «Polonais » qui est un cliché du discours nationaliste, imposé par l'extrême droite dans l'entre-deux-guerres, institutionnalisé par l'occupant nazi et suggérant que ces deux catégories étaient exclusives l'une de l'autre. Je me sers aussi de cette étiquette pour me rapprocher du point de vue des auteur(e)s des textes analysés, ce qui n'exclut pas, bien entendu, une approche critique de leurs discours. Les connotations péjoratives parfois attachées à ce mot ne sont pas non plus étrangères à son choix, comme on le verra dans la suite de mon propos.

6 Ronen 2007; Famulska-Ciesielska 2011.

7 Kichelewski 2018b: 475. 
ou moins précisément (souvent par rapport à des localités plus connues) et parfois brièvement décrits. La danseuse sur un nuage de Regina-Sylvia Cykiert s'ouvre sur la généalogie de l'auteure. C'est à cette occasion qu'y sont évoqués les lieux de naissance de ses ancêtres. Le mode de présence des toponymes ou d'autres mots polonais chez Cykiert, et la plupart des autres auteur(e)s, est toutefois particulier : ils sont souvent déformés. L'on a ainsi « Warki » à la place de «Warka », «Zawierce» au lieu de «Zawiercie », « Wloklawek » et non « Włocławek », « Ozorkov» et non « Ozorków ${ }^{8} »$. On remarque une négligence à l'égard de l'orthographe polonaise qui ressemble à celle constatée par Dorota Głowacka chez Claude Lanzmann et ainsi caractérisée : « [...] les noms des localités polonaises ont été 'massacrés' [...]. Sans parler de l'absence de signes diacritiques, les noms propres polonais sont bizarrement déformés ${ }^{9} »$. Głowacka interprète ces déformations - qui ne concernent que le polonais - comme une sorte de « vengeance linguistique » sur les témoins polonais de l'anéantissement des habitants juifs des localités évoquées. Si Cykiert partage avec Lanzmann cette attitude négligente à l'égard de l'orthographe polonaise, je l'interpréterais plutôt comme une sorte d' ' orientalisme linguistique » ou d'attitude décomplexée par rapport à une langue dominée de la part de locuteurs et locutrices d'une langue dominante ${ }^{10}$. L'orthographe polonaise est allègrement francisée p.ex. par la suppression des signes diacritiques ou l'utilisation du 《V » à la place du « w ». Cykiert ne nourrit d'ailleurs aucun ressentiment à l'égard de la Pologne et a souvent entendu parler non seulement yiddish, mais aussi polonais, dans sa famille. Elle va jusqu'à se demander : « Et pourquoi ne serais-je pas polonaise, moi ? Là sont mes vraies racines, non $?^{11} »$. Cette reconnaissance de sa part polonaise ne va pourtant pas de pair avec l'apprentissage du polonais ni le respect de ses particularités orthographiques, mais elle rend peu plausible l'interprétation en termes de « vengeance linguistique ». En raison du statut subordonné dont jouit forcément le polonais dans des textes écrits et publiés en français, le recours à la théorie de l'orientalisme et des rapports hiérarchiques entre les langues me semble mieux rendre compte des fréquentes erreurs d'orthographe dans les toponymes polonais également chez les autres auteur(e)s.

Dans le livre Wanda. De la Sibérie à Anvers, le courage de la différence de Jerzy Hildebrand, cette orthographe est également souvent fautive : « Rovno » au lieu de « Równe », «Lvov » pour « Lwów », «Zdowbunow » pour « Zdołbunów », « Posnan » à la place de « Poznań », « Kelce » pour « Kielce ${ }^{12} »$... . Si elles témoignant des rapports hiérarchiques entre les langues, ces déformations orthographiques auxquelles l'on a affaire dans quasiment tous les textes et qui ne concernent pas uniquement les toponymes, ne s'accompagnent pas nécessairement d'informations erronées concernant les autres éléments de la réalité polonaise. Au contraire, Jerzy Hildebrand fournit à ses lecteurs et lectrices francophones beaucoup de renseignements justes sur la géographie et l'histoire polonaises au $\mathrm{XX}^{\mathrm{e}}$ siècle, et notamment sur les frontières changeantes de la Pologne.

\footnotetext{
${ }^{8}$ Cykiert 2013:17-18. Dans le texte qui suit, j'adopte l'orthographe polonaise des toponymes, mais dans les citations, je garde les formes utilisées par les auteur(e)s.

9 Głowacka 2016: 305. Sauf mention contraire, je donne toutes les citations des travaux non francophones dans ma traduction.

${ }^{10}$ Je fais ici référence à l'analyse de la situation linguistique mondiale en termes de domination, proposée par Pascale Casanova (Casanova 2002).

11 Cykiert 2013: 79.

12 Hildebrand 2013, respectivement: 6, 19, 20, 55, 68.
} 
Il explique notamment que la partie de l'Ukraine où se trouvait Równe, qu'il habitait avec ses parents, était polonaise à l'époque. Au moment de son retour de Sibérie, et plus précisément du Kazakhstan du Nord, où il a été déporté avec sa mère, il précise que "Les accords de Yalta ont déplacé la frontière orientale de la Pologne vers l'ouest ${ }^{13}$ ». La Pologne apparaît ainsi dans son récit telle une entité politique géographiquement instable, au gré des invasions nazie et soviétique et des accords internationaux mettant fin au deuxième conflit mondial. L'évocation de la géographie polonaise s'avère inséparable de celle de l'histoire du pays. Lorsque le héros-narrateur parle de Varsovie où son oncle paternel a survécu pendant l'occupation allemande en se cachant avec sa famille, il évoque aussi l'insurrection de 1944 contre l'occupant et l'inaction de l'Armée rouge qui, s'étant arrêtée de l'autre côté de la Vistule, contemple l'écrasement du soulèvement par les nazis. Chez d'autres auteur(e)s, les indications géographiques sont aussi accompagnées d'informations historiques ou culturelles. À titre d'exemple, à l'occasion de l'évocation de Góra Kalwaria, chez Adolphe Nysenholc, il est non seulement précisé qu'il s'agit d'une localité " près de Varsovie », mais également que c'était « une capitale du hassidisme $e^{14} »$; des renseignements historiques sur les circonstances de la destruction de la communauté juive de la ville sont aussi ajoutés. L'évocation d'un espace étant indissociable de celle d'un temps, c'est un véritable chronotope de la Pologne qui se met ainsi en place chez de nombreux auteurs.

La présentation chronotopique semble presque naturellement s'imposer dans le cas de la Pologne dans la mesure où ses frontières ont énormément changé au fil de l'histoire, ce nom a donc pu renvoyer à des lieux très divers. Or, la notion de chronotope fait justement référence à « la corrélation essentielle des rapports spatio-temporels » ou «l'indissolubilité de l'espace et du temps ${ }^{15}$ ». L'histoire de la Pologne illustre parfaitement cette constatation de Bakhtine selon laquelle le temps est comme une « quatrième dimension de l'espace ${ }^{16} »$. Ainsi, au moment des invasions nazie et soviétique, en 1939, l'espace polonais cesse-t-il d'être officiellement polonais, ce dont rend compte d'une manière originale le narrateur de Bubelè :

La Pologne occupée par Frank, son gouverneur nazi, était devenue, cruelle ironie, 'Frankreich', l'empire de l'homme franc, homme libre, affranchi, et dont le sport favori [...] était la chasse aux enfants, qu'il tirait comme des lapins, quand ils essayaient de passer sous le mur du ghetto, poussés par la faim, en creusant un trou, leur trou ! Varsovie détruite, ce Gauleiter rétablit Cracovie, la rivale, dans son statut antérieur de capitale, à cinquante kilomètres d'Auschwitz. Toutes villes reliées par la Vistule qui traversait la Pologne de son cours inexorable. Les cendres de Birkenau [...] furent un moment déversées dans ce fleuve, qui longeait en partie le camp. Peut-être celles de ma mère ont été emportées dans ses eaux mêlées jusqu'à Gora Kalwarja ${ }^{17}$.

La Pologne occupée est désignée par des périphrases ironiques : "Frankreich » et « empire de l'homme franc, homme libre, affranchi » qui constituent un jeu de mots à partir du nom de Hans Frank, chef du Gouvernement général, entité créée par les

\footnotetext{
13 Ibid: 55.

${ }^{14}$ Nysenholc 2007: 51.

15 Bakhtine 2013: 237.

${ }_{16}$ Ibid.

${ }^{17}$ Nysenholc 2007: 61.
} 
Allemands d'une partie des territoires polonais occupés, de la signification du mot « franc » en français et de l'appellation allemande de la France. Cette désignation, transposée à la Pologne sur la base du nom de son gouverneur nazi, donne lieu à l'évocation des exactions de ce dernier, vraisemblablement inspirée d'une scène de Kaputt de Curzio Malaparte ${ }^{18}$. Mais les lecteurs et lectrices obtiennent surtout, dans un raccourci, des informations sur l'occupation allemande de la Pologne : le transfert de la capitale à Cracovie ou encore le fait que les cendres des prisonniers gazés et brûlés dans le camp de Birkenau étaient déversées dans la Vistule. Ces renseignements historiques sont donnés dans un style ironique. L'hypothèse des cendres de la mère du héros-narrateur transportées par la Vistule jusqu'à sa ville natale introduit une tonalité macabre. La Pologne occupée devient un locus horribilis en tant que territoire où a eu lieu la Shoah. Dans la mesure où la plupart des textes se réfèrent à cette dernière, ils font aussi appel au topos de la Pologne comme « immense cimetière » dont est fortement imprégnée notamment la littérature israélienne ${ }^{19}$.

\section{Entre locus horribilis et locus amoenus}

La présentation chronotopique pourrait suggérer que c'est la situation historique qui fait de la Pologne un locus horribilis. Mais l'histoire apparaît dans les textes du corpus surtout comme œuvre humaine et c'est le facteur anthropologique qui s'avère décisif dans la construction de ce topos. Pour cette raison, plutôt que de locus horribilis, il vaudrait peut-être mieux parler de populus horribilis. Et ce ne sont pas uniquement les occupants allemands, mais également les Polonais goyim, qui transforment la Pologne en un lieu proprement invivable pour les Juifs. C'est ainsi que la présentation du pays comme locus horribilis n'est pas uniquement liée à l'évocation de la Shoah; elle apparaît également par rapport aux conditions de vie des Juifs polonais aussi bien avant qu'après l'Holocauste. Charles Lerner désigne la Pologne, que ses parents ont quittée dans l'entre-deux-guerres, par l'expression « enfer perdu ${ }^{20} »$. Il inverse ainsi le topos du « paradis perdu ». Même le shtetl, pour lequel il reconnaît que les émigré(e)s - notamment sa mère - nourrissaient une nostalgie, se transforme chez lui en « obligatoire et malheureuse réponse aux antisémitismes, à la profanation, à l'ignorance... ${ }^{21} »$. Le shtetl polonais est pour lui un ghetto ${ }^{22}$ alors que ce dernier terme désigne normalement un quartier juif et non une localité à majorité juive (comme le shtetl) et qu'il charrie, surtout depuis la Shoah, des connotations extrêmement négatives. En revanche, c'est par rapport au quartier juif de Liège que Lerner utilise finalement le mot «shtetl» (orthographié « stetl »), comme si ce vocable aux connotations positives ne pouvait s'appliquer qu'à la réalité belge alors qu'il désignait normalement les localités juives d'Europe centrale et

${ }^{18}$ Il s'agit d'une scène dans laquelle le protagoniste de Kaputt accompagne Frank dans les rues de Varsovie et ce dernier tire sur des enfants qui essaient de passer sous le mur du ghetto.

19 Famulska-Ciesielska 2011.

20 Lerner 2007: 23.

21 Ibid.: 23.

${ }^{22}$ Le mot « ghetto » comme synonyme du « shtetl » apparaît aussi dans la pièce d'Anna Stelkowicz. 
orientale ${ }^{23}$. Tout ce qui est lié au pays d'origine des parents est négativisé, ce qui crée une contradiction dans le récit de Lerner dans la mesure où il parle aussi de leurs tentatives de recréer le shtetl de leur enfance en Belgique. Comment expliquer cette nostalgie de l' « enfer perdu » chez des immigré(e)s qui se retrouvent, selon l'auteur, « dans un pays moderne, sans ségrégations ${ }^{24} »$ ? En utilisant une expression qui fait immédiatement penser au « paradis perdu », Lerner semble lui-même reconnaître implicitement que le rapport des émigrés juifs à la Pologne était plus positif que ne le laisse entendre son analyse explicite. Il fait pourtant tout pour négativiser le pays et le transformer en un locus horribilis avec lequel contraste la présentation de la Belgique comme « Eldorado ${ }^{25}$ ». Il parle ainsi d' « une méchante vague d'antisémitisme » qui régnerait en Pologne « depuis trois siècles ${ }^{26} »$, faisant le pont entre le $\mathrm{XVII}^{\mathrm{e}}$, avec les massacres des Juifs perpétrés par les Cosaques, et l'antisémitisme du début du $\mathrm{XX}^{\mathrm{e}}$. Cette analyse historique reliant deux époques très différentes s'inscrit parfaitement dans la construction d'un locus horribilis polonais à l'aide de l'évocation de l'antisémitisme des habitants du pays. Un contraste entre ce dernier et un locus amoenus belge existe non seulement chez Lerner, mais aussi dans nombre d'autres textes, y compris dans Monsieur Optimiste d'Alain Berenboom dont les parents ne cessent d'opposer la Belgique de leurs rêves à une Pologne honnie.

À côté du chronotope de la Pologne comme locus horribilis par excellence, apparaît celui du locus amoenus de l'enfance. Même Lerner reconnaît que, pour sa mère, le shtetl polonais de son enfance constituait « l'image d'un bonheur intransmissible ${ }^{27}$ », une sorte d'archétype de la félicité. Une image proche est présente aussi dans L'apprentissage inutile de David Scheinert. L'incipit du roman est construit à partir de perceptions sensorielles, surtout visuelles, du héros-narrateur. Celui-ci se remémore notamment des bruits et des images du paysage polonais. Il s'agit d'une collection de perceptions sans autre lien entre elles que celui qui perçoit : coups de feu, neige, arbres, myrtilles, cris, chaleur et douceur du corps de sa nounou, goût des pâtisseries... Avant que ce qui l'entoure puisse former un tout cohérent, le petit garçon part avec ses parents pour la Belgique. La description de cette série d'éléments disparates qui correspondent dans son esprit à la Pologne laisse toutefois une impression euphorique, fruit de la sensualité des images. Conformément à la tradition, la nature joue un rôle important dans ce locus amoenus qui embrasse l'ensemble de la réalité environnante que le petit enfant découvre avec tous ses sens. La narration est ici tissée de ces «stimuli sensoriels [...] conservés dans la mémoire et $[. .$.$] associés à des sentiments » que Karolina Famulska-Ciesielska énu-$ mère parmi les principales composantes de la « vision mentale » de la Pologne chez les écrivains polono-israéliens : images, parfums, couleurs ${ }^{28}$... Une Pologne campagnarde, blanche, ensoleillée et à l'horizon illimité s'oppose d'ailleurs chez Scheinert à une Belgique urbaine, sombre, identifiée à « une grande gare et une ville très noire » où les personnages habitent « dans une rue étroite, sale et qui sent mauvais ${ }^{29}$ ». La Pologne correspond ici à la découverte émerveillée de la beauté du monde, la Belgique - à celle de

\footnotetext{
${ }^{23}$ Cała, Węgrzynek, Zalewska 2000: 97; 338.

24 Lerner 2007: 24.

25 Ibid.: 14.

26 Ibid.: 10.

27 Ibid.: 17.

28 Famulska-Ciesielska 2011.

29 Scheinert 1985: 13-14.
} 
sa laideur. Eva et Rivka, personnages de la pièce Eva, Rachel, Yitzhak et les autres... De l'émigration polonaise à l'enfant caché d'Anna Stelkowicz, se souviennent aussi d'une nature polonaise idyllique. De la même manière que Natek, héros de la nouvelle Les immigrés du recueil L'immigré Herschel Schaerbeeker raconte de Zalek Kalb Beller, qui oppose sa « verte Galicie » et les « grandioses montagnes enneigées de son pays ${ }^{30}$ » à la noirceur des corons belges. Une Pologne au paysage naturellement beau contraste à nouveau avec une Belgique défigurée par l'industrialisation. La perspective s'inverse : c'est cette dernière qui est un locus horribilis, et cette première, un locus amoenus.

Une autre variante du locus amoenus polonais est liée à l'évocation de la vie de la communauté juive traditionnelle. Ainsi, dans Une adolescence perdue dans la nuit des camps, Henri Kichka évoque-t-il des souvenirs nostalgiques du shtetl de ses parents. L'un des signes de cette nostalgie est le maintien dans la communauté immigrée des deux langues principales parlées au quotidien par les Juifs polonais : le yiddish et le polonais. Regina-Sylvia Cykiert l'évoque dans La danseuse sur un nuage, Alain Berenboom dans Monsieur Optimiste. Dans le livre de Jerzy Hildebrand, après s'être installés à Anvers, Wanda et Abraham, continuent aussi à communiquer en polonais à la maison. Wanda vit dans la nostalgie de « [s]a Varsovie d'avant la guerre ${ }^{31}$ » où elle menait une riche vie sociale. Sa nostalgie est celle des Juifs polonophones et s'identifiant à la culture polonaise, mais dans la majorité des textes, il s'agit de celle du « yiddishland ${ }^{32} »$, un monde juif yiddishophone, vivant sa vie d'une manière relativement autonome dans ce pays multiethnique, multiconfessionnel et multilingue que fut la Pologne d'avant la Seconde Guerre mondiale. La nostalgie est aussi associée à la vie familiale, comme pour Natek, héros de la nouvelle précitée dont le narrateur nous dit que : «[...] les images simples et douces de la vie familiale l'obsèdent : le couvert dressé pour le sabbat avec les chandeliers portant les bougies allumées, sa mère en habits de fête, son père ravi dans sa souquenille reprisée et luisante ${ }^{33} \ldots$.. . La vie de famille apparaît ici dans son aspect festif, lié à l'observance des traditions religieuses du judaïsme, en l'occurrence la célébration du sabbat. Mais, tout de suite après, surgit dans l'esprit du personnage une autre image, celle « de la misère noire, de la lutte incessante pour vaincre la faim, des petits frères et sœurs qui vont pieds-nus ${ }^{34} \gg$. Le locus amoenus et le locus horribilis alternent dans ses souvenirs, comme deux faces d'une même réalité, ce qui dote cette dernière d'une coloration émotionnelle ambivalente. Néanmoins, le locus amoenus reste fortement lié à l'évocation d'un espace (géographique et culturel), quand le locus horribilis est surtout associé à l'environnement humain goy.

\footnotetext{
30 Kalb Beller 1978: 33.

31 Hildebrand 2013: 114.

32 Skowronek 2017: 29; Berenboom 2013: 107.

33 Kalb Beller 1978: 25-26.

34 Ibid.: 26.
} 


\section{Une Pologne « autre »}

Qu'elle soit locus amoenus ou locus horribilis, la Pologne est, dans la plupart des textes, un ailleurs qui apparaît irrémédiablement autre. On le voit clairement dans cette scène de L'apprentissage inutile :

[...] nous nous arrêtâmes, la main dans la main, derrière un attroupement. Les gens lisaient une affiche. Ordre de mobilisation. Ah oui, il n'y avait pas que nous deux... L'Allemagne avait envahi la Pologne... La France et l'Angleterre lui déclaraient la guerre.... Que c'était bête... Ici, on se tenait par la main, on sentait la vibration de son corps, on s'étreignait, parce qu'on était jeunes, parce qu'on voulait se plaire, jouir de soi, de l'autre, du soleil, du sable. Ailleurs, il y avait un autre monde avec d'autres lois ${ }^{35}$.

Le héros-narrateur, coupé du monde car accaparé par ses amours, est ramené à la réalité par une affiche de mobilisation au moment de l'éclatement de la Seconde Guerre mondiale. La Pologne est ici un locus horribilis, un ailleurs menaçant par rapport à la Belgique des amours juvéniles. Elle devient l'incarnation géographique de la dure réalité avec ses lois implacables. C'est « un autre monde » qui fait irruption dans l'idylle amoureuse que le protagoniste vit en bord de mer avec Else Besserding, femme d'un réfugié juif autrichien, et en marque la fin. Aux immigrés juifs polonais, la Pologne apparaît donc surtout par contraste avec leur pays d'accueil, comme on l'a déjà vu en évoquant l'opposition entre locus amoenus et locus horribilis, alternativement incarnés par l'un ou l'autre pays.

Dans l'essai de René Kalisky « Belgique ? Le pays le plus imaginaire du monde », la Pologne est également évoquée par comparaison à la Belgique, toujours dans un esprit d'opposition bien que l'auteur commence son raisonnement en signalant une ressemblance entre les deux pays : il les voit tous les deux comme des pays-frontières. Il les définit avant tout par leur situation géographique, déterminante pour leur histoire :

La Belgique est [...] par excellence le pays d'entre-deux. Elle se trouve dans une situation qui ne peut se comparer qu'à celle de la Pologne... Une Pologne dont on finit par nier le droit à la vie, puis à la survie, en même temps que se développent de part et d'autre de ses frontières tantôt politiques, tantôt naturelles, mais toujours contestées, les puissances concurrentes de la Prusse et de la Russie. Mais la Pologne résiste ; qu'on la dépèce, la partage, la rapièce, la ravaude, elle résiste. La Pologne homogène résiste. La Pologne hétérogène résiste. Elle tient bon, renaît de ses cendres quels que soient les avatars que lui réserve son destin. La situation de pays d'entre-deux de la Pologne n'a jamais prévalu sur le sentiment national de son ou de ses peuples. Aux pires moments de leur histoire, les Polonais refusent la déshistoire, ce qui leur évite d'avoir à combattre l'idée de la désexistence et ses démons ${ }^{36}$.

Kalisky commence par une similitude due à la situation géographique des deux pays, situés entre des voisins puissants : la Russie et l'Allemagne pour la Pologne, l'Allemagne et la France pour la Belgique. Mais il aboutit à un contraste : alors qu'en Belgique, selon lui, la « mode $[\ldots]$ est $[\ldots]$ à la négation de sa propre histoire, à une entreprise de déshistoire » qui «ne peut déboucher que sur la désexistence : le renoncement

\footnotetext{
35 Scheinert 1985: 146-147.

36 Kalisky 2006: 300.
} 
à soi-même ; le divorce [...] des communautés qui se partagent le pays ${ }^{37} »$, il ne voit rien de tel en Pologne. À une Belgique menacée d'éclatement, Kalisky oppose donc finalement une Pologne sûre de son existence, malgré une situation géographique tout aussi problématique et qui a abouti au partage du territoire polonais entre ses voisins, à la fin du XVIII' siècle. Il attribue cette différence à l'attitude des Polonais envers l'histoire : sa célébration leur confierait leur identité alors que les Belges qui lui tourneraient le dos se trouveraient menacés de désexistence. Kalisky montre donc aux Belges le peuple polonais comme modèle d'un rapport positif à l'histoire, constitutif d'une identité nationale forte. À une identité belge évanescente, il oppose une identité polonaise puisant sa force dans le passé. Sous sa plume, la Pologne devient un pays à la fois proche de la Belgique (par sa position géographique) et un contre-modèle positif (par son climat idéologique) dans lequel il serait possible de puiser un remède contre les problèmes communautaires agitant le plat pays. Elle est une sorte de reflet inversé.

\section{Une vision passéiste de la Pologne}

Dans les œuvres du corpus, la Pologne est le lieu des origines des immigré(e)s ou de leurs ancêtres, c'est donc celle du passé qui est le plus souvent évoquée. Il s'agit alors d'un portrait mémoriel, ancré dans les souvenirs et qui, dans la mesure où il se réfère aux origines, a une dimension mythique ${ }^{38}$. Du point de vue narratif, il est lié à une poétique de la mémoire et apparaît dans des segments analeptiques. Le narrateur second du Livre de Joseph de Bernard Dan, c'est-à-dire Joseph lui-même, ancêtre supposé du narrateur premier, évoque ainsi, dans un texte censé être écrit quand l'insurrection du ghetto touche à sa fin, « la grande, la belle, la juive et craignant-le-Nom Varsovie ${ }^{39}$ ", à savoir celle qui vient d'être anéantie sous ses yeux. Comme nous l'avons déjà constaté à propos de l'image de la Pologne en tant que locus horribilis, dans l'évocation du pays, le rôle définitoire est souvent joué par une référence à cette rupture décisive dans l'histoire des Juifs polonais que fut la Shoah. Par conséquent, le narrateur premier du Livre de Joseph, Jean-Paul Rakover, définit la Pologne à la fois comme « le berceau de [s]es ancêtres » et «l'urne funéraire vide des cendres de [s]on grand-père et de sa famille $e^{40} »$. Le pays est donc métaphoriquement désigné par deux objets dont l'un symbolise le début de la vie (le berceau du nouveau-né), l'autre, sa fin (l'urne funéraire). Cette double désignation métaphorique recèle une nouvelle ambivalence - d'espace originel, la Pologne est susceptible de se transformer en dernière demeure. Elle est espace de vie, mais aussi de mort ; berceau et tombeau.

Dans le scénario du film Bruxelles-transit de Samy Szlingerbaum, qui raconte l'émigration d'une famille juive polonaise en Belgique, au lendemain de la Seconde Guerre mondiale, la représentation de la Pologne est encore plus fortement associée au passé

\footnotetext{
37 Ibid: : 299-300.

38 Shoshana Ronen remarque cette même dimension chez les écrivains israéliens parlant de la Pologne (voir Ronen 2007: 11-12).

39 Dan 2014: 153.

40 Ibid.: 132.
} 
et la Shoah pèse encore plus lourdement sur elle. Au début, une voix féminine parle du voyage entre Łódź et Paris, en passant par Varsovie et Prague. Les images doivent toutefois montrer Bruxelles, et notamment la gare du Midi. La voix évoque donc p.ex. Varsovie : « Nous sommes arrivés à Varsovie, là... tout était démoli... ${ }^{41}$ » mais ce sont des images de la capitale belge qui doivent « illustrer », ou plutôt accompagner, son récit. La Pologne est évoquée sans être représentée. Elle reste uniquement présente dans le souvenir de la femme qui parle. Par ailleurs, le scénariste la définit, dans le commentaire de cette séquence, comme «terre-musée de l'horreur ${ }^{42} »$. Il effectue ainsi une «muséification » du pays, la Pologne devenant surtout un espace de conservation des traces de la Shoah. Un peu plus loin, il parle de Łódź et de Varsovie en tant que « villes évanouies ou sacrifiées ${ }^{43} »$, comme si elles avaient complètement disparu avec leurs habitants juifs.

Cette façon de (ne pas) représenter la Pologne vient de son statut paradoxal dans l'esprit du scénariste qui, pendant longtemps, en a entendu parler, sans en avoir une expérience directe. Il explique qu'il a finalement visité la ville de Kazimierz d'où était originaire la famille de sa mère et où il « connaissai $[\mathrm{t}]$ toutes les maisons » car « $[\mathrm{S}]$ on enfance, [il] l'[a] vécue à Kazimierz, à travers les récits de [s]a mère ${ }^{44} \gg$. Jusqu'à cette visite, le récit maternel restait pour lui le seul support de l'existence de la Pologne. Cette dernière était un (non-)lieu entièrement narratif, fait de paroles et identifié au passé familial. Il lui en restait une seule image, une photo de la maison de ses grands-parents à Kazimierz, envoyée avant la guerre à des membres de la famille résidant à l'étranger. Le scénariste l'appelle « le seul souvenir du monde d'avant, passé sacré intouchable ${ }^{45}$ ». Chez Szlingerbaum, la Pologne d'aujourd'hui semble frappée d'un interdit de représentation. Et celle du passé est évoquée dans une langue indissolublement liée à la vie juive polonaise, presque entièrement anéantie : le yiddish que doit parler la femme dans le film. C'est cette langue qui semble devenir une métonymie de la Pologne juive du passé, à jamais perdue. Selon Lilly Scherr, « La langue symbolise cet 'avant', qui ne peut être dit ni montré $[. .$.$] . Elle devient le seul territoire... { }^{46} »$. Mais, en réalité, cet « avant » est constamment dit, il doit même être momentanément montré, toutefois, justement à travers une image qui vient d'alors : la vieille photo. Malgré la visite effectuée, la seule image possible de la Pologne reste celle immortalisée sur la photo d'avant la catastrophe de la Shoah. Comme l'explique le scénariste, la maison familiale qui y est immortalisée, a été donnée à des paysans polonais qui avaient caché sa famille pendant la guerre. C'était « le tribut payé pour la survie ${ }^{47} »$. Il ne peut donc plus la considérer sienne. Elle appartient à un passé irrécupérable, comme le pays lui-même.

La façon dont Samy Szlingerbaum (ne) représente (pas) la Pologne, illustre très bien son mode d'existence dans l'esprit de nombreux descendants des Juifs polonais. Elle est un lieu surgissant uniquement des souvenirs des parents ou grands-parents. Comme le formule Lydia Flem, c'est « un ailleurs sans images ${ }^{48}$ », celui du passé familial qui

\footnotetext{
${ }^{41}$ Szlingerbaum 1989: 26.

42 Ibid.: 27.

43 Ibid.: 29.

44 Ibid.: 31.

45 Ibid.: 30.

46 Scherr 1989: 80.

47 Szlingerbaum 1989: 30.

48 Flem 1989: 94.
} 
a déterminé leur destin mais qu'ils ont du mal à s'approprier, même après l'avoir visité, car il n'est plus le même que dans les souvenirs qui leur ont été transmis. La Pologne d'aujourd'hui n'est pas totalement absente des textes, mais elle semble toujours convoquée en fonction du passé. Dans Le livre de Joseph, le héros-narrateur, qui y vient pour un congrès scientifique, reconnaît que « Varsovie est déjà devenue une ville de congrès. La Pologne est libre. Elle fait partie de l'Union européenne avec la France et l'Allemagne, toutes amies et démocratiques ${ }^{49} »$. De cette Varsovie moderne, il mentionne pourtant surtout les lieux de mémoire visités : vestiges du mur du ghetto, monument à ses héros, musée Pawiak. Même s'il parle de la Pologne d'aujourd'hui, il le fait avant tout pour évoquer des traces de son passé juif. Il en va de même dans de nombreux autres textes.

\section{Le portrait des Polonais goyim et la question de la stéréotypisation}

Comme on l'a déjà vu à propos du lien entre locus horribilis et populus horribilis, le portrait de ses habitants fait partie intégrante de l'image du pays. Les Polonais goyim sont habituellement soit représentés sous les espèces d'un personnage collectif et désignés au pluriel, soit individualisés, mais anonymes. Ce sont le plus souvent des personnages secondaires, des «figurants », et leurs apparitions restent brèves. Ils appartiennent à l'arrière-plan des histoires racontées. Ils sont des personnages "plats », selon la formule d'E. M. Forster ${ }^{50}$, pourvus d'une caractérisation rudimentaire. Celle-ci est réductionniste : un petit nombre de traits leur est attribué. Leur étiquette se réduit d'ordinaire à la mention de leur nationalité. Qui plus est, dans de nombreuses œuvres du corpus, l'on remarque la reprise d'une conception oppositionnelle entre « Juif » et « Polonais », promue par les discours nationalistes. Les Polonais goyim sont couramment désignés simplement comme « Polonais », par opposition aux « Juifs », même à propos des périodes où ces derniers étaient des citoyens polonais. Tel fut évidemment le cas en Pologne pendant des siècles, y compris dans l'entre-deux-guerres, quand le pays comptait plus de 3000000 de citoyens de confession juive. Mais, c'est justement à cette époque que l'extrême droite polonaise les excluait systématiquement de la communauté nationale et le mouvement sioniste les considérait aussi comme une nation séparée ${ }^{51}$, en dépit de leur nationalité. La « judéité » et la « polonité » étaient alors assez couramment opposées l'une à l'autre.

Dans le récit de sa déportation, après l'invasion russe de l'est de la Pologne, en septembre 1939, Jerzy Hildebrand montre l'exemple d'une famille pour laquelle la distinction entre « Juifs » et « Polonais » n'a au départ aucun sens. Dans l'entre-deux-guerres, ses membres se sentent polonais, sans y voir de contradiction avec leurs origines confessionnelles. Hildebrand rend aussi compte d'une communauté de destins entre Polonais juifs et goyim appartenant à la même classe sociale, sous le régime soviétique : ils formaient le groupe de «bourgeois polonais déportés [...] pour 'bénéficier' d'une

\footnotetext{
49 Dan 2014: 116.

50 Bordas, Barel-Moisan, Bonnet, Déruelle, Marcandier 2015: 162.

51 Il faut pourtant rappeler que le sionisme est né principalement en réaction à l'antisémitisme (notamment à l'affaire Dreyfus en France).
} 
rééducation prolétarienne ${ }^{52} »$. L'auteur-narrateur présente ses parents comme « issus de la petite bourgeoisie juive, déjà fort assimilée ${ }^{53} »$, ne parlant pas yiddish et ne pratiquant pas le judaïsme. Selon lui, ils étaient « profondément attachés à la Pologne, à sa langue et à sa culture et ne $\mathrm{p}[\mathrm{ou}] \mathrm{v}$ [ai] ent envisager de vivre ailleurs ${ }^{54} »$. Le père du narrateur, officier de réserve, mobilisé en 1939, fait prisonnier par les Soviétiques, sera d'ailleurs assassiné à Katyń avec des milliers de ses collègues d'autres confessions. Une fois déportée, sa mère, Wanda, acceptera la citoyenneté soviétique seulement sous la contrainte. Après la guerre, elle décidera de rentrer en Pologne avec son fils. Malgré l'insistance du narrateur sur les sentiments patriotiques de ses parents et leur polonité, après leur retour de déportation, Jerzy et sa mère se rendent vite compte qu'ils ne sont pas les bienvenus dans la Pologne de l'après-guerre. D'après Hildebrand, sa famille « faisai[ $\mathrm{t}$ ] partie de ces Juifs qui, selon l'expression de Sartre, ne le sont que parce que les autres les reconnaissent comme tels ${ }^{55} »$. En effet, c'est seulement dans le regard des Polonais goyim que le petit Jerzy se découvre Juif. Une femme rencontrée dans un tramway, à Poznań, lui demande sur le mode de l'alternative : «Et toi, petit, tu es polonais ou juif ? ${ }^{56}$ ", comme s'il s'agissait de deux identités exclusives l'une de l'autre. En déportation, le destin commun des Polonais juifs et goyim a, dans une large mesure, effacé l'opposition nationaliste entre « Juifs» et « Polonais », mais dès que Jerzy et Wanda se retrouvent en Pologne, cette dichotomie surgit dans le récit. Les Polonais goyim imposent aux personnages une identité juive s'opposant à l'identité polonaise. Apparaît ainsi la caractéristique idéologique qui leur est le plus communément attribuée dans les œuvres du corpus : leur antisémitisme, se manifestant en l'occurrence par l'exclusion des Juifs de la communauté nationale. Alina Cała voit justement dans le fait de considérer les Juifs comme des étrangers, quand bien même ils résideraient depuis des siècles sur un territoire, l'une des principales manifestations d'antisémitisme ${ }^{57}$. Illustrant le propos de Sartre, dans Réflexions sur la question juive, c'est donc l'expérience de l'antisémitisme qui devient constitutive pour Wanda et Jerzy d'une identité juive comprise comme s'opposant à l'identité polonaise choisie au départ.

Le portrait des Polonais goyim a ainsi surtout un caractère relationnel. Ils sont définis en priorité par leur attitude (hostile) envers leurs concitoyens juifs. Si l'on voulait analyser les histoires racontées dans les œuvres du corpus en termes actantiels, les Polonais goyim y apparaîtraient le plus souvent en position d'opposants. Qu'il s'agisse de textes qui parlent de l'émigration ou de ceux relatifs à la Shoah, ils sont habituellement des antagonistes des sujets juifs luttant pour une vie meilleure ou, tout simplement, pour la survie. C'est surtout le cas lorsqu'ils apparaissent en tant que personnage collectif, foule anonyme hostile. En revanche, comme figures individualisées, ils jouent parfois le rôle d'adjuvants. Dans Écris, papa, écris, Elie Rozencwajg décrit des relations de voisinage amicales de ses parents avec leurs voisins polonais chrétiens, basées notamment sur l'entraide. Le héros-narrateur vient ainsi au monde notamment grâce à l'aide d'une

\footnotetext{
52 Hildebrand 2013: 6.

53 Ibid.: 19.

54 Ibid.: 22.

55 Ibid.: 85.

56 Ibid.: 56.

57 Cała, Węgrzynek, Zalewska 2000: 9.
} 
voisine chrétienne, sage-femme expérimentée. Dans Plus que la vie de Charles Lerner, Aron, l'oncle de l'auteur, est prévenu par « le concierge de son immeuble - un Polonais non juif ${ }^{58} \gg$ d'un pogrome qui se prépare. Dans Témoignage de Sylvain Gutmacher, où le portrait collectif des Polonais goyim détenus à Auschwitz est nettement péjoratif, apparaît un portrait individuel beaucoup plus positif, celui de Marek, un Blockältester (doyen du bloc), dur, mais juste et animé d'un esprit de solidarité, que les autres prisonniers voient tel « un homme comme eux $^{59}$ » et non un adjuvant des tortionnaires nazis.

En dépit de ces contre-exemples positifs, l'antisémitisme en tant que caractéristique majeure des Polonais goyim apparaît dans la majorité des textes, surtout à l'occasion de l'évocation des raisons de l'émigration des familles juives. On y voit clairement que nombre d'émigrés juifs de l'entre-deux-guerres, mais également d'après-guerre, se sentaient poussés hors de Pologne par le climat idéologique qui y régnait et les actes de violence qu'il inspirait. Même si parfois des motifs économiques de l'émigration transparaissent en filigrane, ils sont passés sous silence ou minimisés et c'est l'explication idéologique qui prédomine. Il en va ainsi dans Deuxième génération de Michel Kichka. L'émigration des ancêtres maternels et paternels du héros est expliquée par l'antisémitisme des Polonais goyim : «Les Swierczynski, tout comme les Kichka, avaient fui l'antisémitisme polonais dans l'entre-deux-guerres ${ }^{60} »$. Son père, Henri Kichka, dans Une adolescence perdue dans la nuit des camps, donne la même explication. Les deux présentent la Pologne comme un endroit d'où il fallait fuir. Michel Kichka précise que son grand-père paternel venait d'une famille de douze enfants ${ }^{61}$, l'on peut donc supposer que des motifs économiques ont également joué un rôle dans son émigration, mais c'est l'antisémitisme qui est retenu comme seul facteur explicatif. Regina-Sylvia Cykiert parle aussi de sa « famille de valeureux Liégeois dont les parents sont venus à pied ou en train de Pologne pour fuir les pogroms ${ }^{62} »$. Dans la nouvelle Jenny du recueil de Zalek Kalb Beller, apparaît également une famille juive polonaise « fuyant les pogroms ${ }^{63}$ ». Nathalie Skowronek cite parmi les motifs qui ont poussé ses ancêtres à quitter la Pologne « sa morosité économique et son antisémitisme ${ }^{64}$ ». Dans la mémoire familiale des Kichka, Cykiert, Skowronek ou Hildebrand, la Pologne reste donc associée à l'antisémitisme de ses habitants non juifs et à leurs actes de violence envers leurs concitoyens de confession juive.

Les études historiques confirment le lien entre le développement de l'antisémitisme (prenant appui sur l'antijudaïsme antérieur, propagé par l'Église), depuis la fin du XIX siècle, résultant de l'essor du mouvement nationaliste polonais, et les vagues successives d'émigration juive ${ }^{65}$. Dans l'entre-deux-guerres, l'on a effectivement affaire en Pologne à une exacerbation et une institutionnalisation des discriminations envers les citoyens de confession juive. À cette époque, selon Daniel Tollet, « Le courant nationaliste polonais répondait aux situations de crise économique par l'antisémitisme actif », et l'émigration,

\footnotetext{
58 Lerner 2007: 61.

59 Gutmacher 2010: 54.

60 Kichka 2012: 28.

61 Ibid.: 73.

${ }^{62}$ Cykiert 2013: 119.

63 Kalb Beller 1978: 59.

64 Skowronek 2017: 27.

65 Voir Schreiber 2004: 231-232, 237.
} 
qui a concerné presque 400000 Juifs polonais, entre 1921 et 1937, était due « à la misère puis à l'antisémitisme ${ }^{66} \gg$. Les mesures discriminatoires se multipliaient, se traduisant p.ex. par la limitation de l'accès des Juifs à l'enseignement supérieur, l'isolement des étudiants juifs (les «bancs de ghetto ») ou le boycott des commerces juifs, mais également par des pogroms. Jerzy Hildebrand fait allusion à l'une de ces discriminations lorsqu'il dit que son père a pu faire des études à l'école polytechnique de Varsovie «malgré un numerus clausus de fait ${ }^{67}$ ». Le narrateur de la nouvelle Les immigrés du recueil de Zalek Kalb Beller mentionne aussi « un numerus clausus rigoureux ${ }^{68}$ » dans les universités polonaises, surtout pour insister sur le manque de perspectives pour la jeunesse juive en Pologne. Haïm, l'un des personnages de la pièce d'Anna Stelkowicz, fait référence aux « bancs de ghetto ». Bien que les raisons de l'émigration aient aussi pu être économiques ou politiques, p.ex. liées au développement des mouvements sioniste ou communiste, c'est justement l'existence d'un climat social antisémite qui s'est gravée dans la mémoire des émigrés juifs polonais. Le héros-narrateur de L'apprentissage inutile de David Scheinert le décrit d'une manière plus indirecte, métaphorique quand il dit : « Mon père avait quitté la Pologne parce qu'il était difficile d'y respirer ${ }^{69}$ ». Dans un style poétique caractéristique de son récit, il renvoie pourtant au même climat social hostile que les autres auteur(e)s.

À propos de la Seconde Guerre mondiale, c'est la collaboration de certains Polonais goyim avec l'occupant nazi et leur participation à la «solution finale » qui sont évoquées. Les travaux historiques confirment, dans une large mesure, la croissance des sentiments antisémites chez les Polonais goyim, sous l'occupation allemande : en font état notamment des rapports officiels adressés au gouvernement polonais exilé à Londres par ses envoyés dans la Pologne occupée ${ }^{70}$. Dans Une adolescence perdue dans la nuit des camps, Henri Kichka raconte la brutalité des contremaîtres polonais qui dirigent les prisonniers juifs travaillant dans le camp de Tarnowitz et les présente comme des complices des nazis. Dans J'avais 16 ans à Auschwitz, Henri Sonnenbluck décrit même les prisonniers polonais du camp d'Auschwitz en tant que « dominateurs et méchants » et tels des « bourreaux 'suppléants' » qui harcèlent leurs codétenus juifs, et le polonais, à côté de l'allemand, est pour lui l'une des langues de « [leurs] tortionnaires ${ }^{71} »$. Dans Témoignage de Sylvain Gutmacher, à Auschwitz, selon le narrateur, les Polonais « étaient les 'caïds' du camp, des capitalistes ${ }^{72}$ », vivant mieux que les autres prisonniers, grâce aux colis qu'ils recevaient et qui leur permettaient de pratiquer le troc. Dans le même texte, apparaissent « des civils polonais, maçons et charretiers » qui, dans les ruines du ghetto de Varsovie, «à la recherche d'objets précieux [...] rivalisent de rapacité avec les $\mathrm{SS}^{73}$ ». Cette image concorde avec les travaux de Jan Tomasz Gross sur les avantages économiques tirés par une partie de la population polonaise du génocide des Juifs ${ }^{74}$. Max

\footnotetext{
66 Tollet 1992: 265, 274.

67 Hildebrand 2013: 19.

68 Kalb Beller 1978: 15.

69 Scheinert 1985: 32.

70 Libionka 2009.

71 Sonnenbluck 1990, respectivement: 43, 48, 83.

72 Gutmacher 2010: 59.

73 Ibid.: 81.

74 Gross 2014.
} 
Gorder, le protagoniste du roman de Gutmacher, retrouve, dans les ruines du ghetto, le journal intime d'une jeune fille juive qui présente clairement parmi les raisons de l'échec du soulèvement du ghetto les promesses d'aide non tenues des " non-Juifs ${ }^{75}$ ", sous-entendu, de la Résistance polonaise ou, plus largement, de la population non juive de Varsovie. Dans Le livre de Joseph de Bernard Dan, Joseph, combattant dans cette même insurrection, raconte la mort de sa fille de dix ans, Rachel, poursuivie par « les sentinelles nazies et des dizaines d'auxiliaires polonais ${ }^{76} »$, après qu'elle a franchi le mur du ghetto à la recherche de la nourriture. Des Polonais y apparaissent clairement comme complices des génocidaires allemands. Mais, en contrepoint, Jean-Paul, narrateur premier du roman, mentionne aussi Irena Sendlerowa, la présentant en tant que celle qui « a sauvé beaucoup d'enfants - deux mille cinq cents ? - pendant la guerre en utilisant toutes sortes de stratagèmes - déguisements, ambulances, cercueils, sacs de pommes de terre $^{77} \gg$. Chez Dan, le portrait des Polonais goyim n'est donc pas univoque - à côté des chmaltsovnik (maîtres-chanteurs et dénonciateurs) apparaît l'une des héroïnes de l'aide aux Juifs. La plupart des autres auteur(e)s parlant de la même époque mentionnent plus souvent des collaborateurs polonais des nazis.

L'antisémitisme est évoqué également à propos de l'après-guerre. Dans Bubelè. L'enfant à l'ombre d'Adolphe Nysenholc, qui raconte le destin d'un enfant né de parents polonais, caché par un couple belge, l'oncle paternel du garçon, Abraham, seul survivant de la famille, s'étant caché à la campagne, vient chercher son neveu après la guerre. Il lui raconte sa tentative de revenir dans la maison familiale, à Góra Kalwaria : « Les Polonais occupaient les maisons 'abandonnées'. On lui conseilla de ne pas entrer dans la sienne, s'il tenait à la vie ${ }^{78} \gg$. Adolphe Nysenholc fait ici état d'une situation qu'ont rencontrée de nombreux survivants de la Shoah. L'historien Daniel Tollet la résume ainsi : « [...] le retour des rescapés juifs dans leurs villes et leurs villages créait une situation conflictuelle grave car des Polonais s'étaient appropriés les appartements et les biens des Juifs $^{79} »$. Se sentant menacé, Abraham quitte la Pologne. Dans Wanda, après leur retour de déportation, l'héroïne éponyme et son fils sont hébergés à Sosnowiec par Joseph, le beau-frère de Wanda, et par sa femme, Sabina. Ces derniers font tout pour cacher leurs origines juives. Jerzy caractérise le climat social général régnant à cette époque de la manière suivante : "Malgré le petit nombre de Juifs polonais qui ont survécu, l'antisémitisme est plus virulent encore qu'avant la guerre. On ne se contente plus de vexations et d'insultes verbales. Plusieurs pogroms éclatent ${ }^{80} \gg$. Il cite le plus connu, celui de Kielce, en juillet 1946, mais les recherches historiques actuelles font état de plus d'une centaine d'incidents de violence antijuive sur le territoire polonais, entre septembre 1944 et septembre $1946^{81}$. Hildebrand rapporte aussi l'histoire d'un cousin de sa mère, renvoyé de l'armée polonaise à cause de ses origines juives. L'antisémitisme ambiant détermine la décision de Wanda d'émigrer. Il s'agit d'une réaction assez courante à la vague de violences qui accompagne le retour des rescapés juifs, selon les recherches

\footnotetext{
75 Gutmacher 2010: 84-85.

76 Dan 2014: 66.

77 Ibid.: 112.

78 Nysenholc 2007: 61.

79 Tollet 1992: 298.

80 Hildebrand 2013: 67-68.

${ }^{81}$ Kichelewski 2018a: 29.
} 
d'Audrey Kichelewski ${ }^{82}$. L'une des formes de cette violence est rapportée par Julek Bar qui, racontant un voyage en train entre Katowice et Varsovie, dit y avoir rencontré un groupe de bandits qui jetaient les Juifs des trains ${ }^{83}$. L'antisémitisme est parfois attribué à une partie de la population polonaise seulement - p.ex. chez Hildebrand, il est contrebalancé par un portrait élogieux des Micinski, famille de catholiques fervents dans laquelle est entrée par mariage une grand-tante maternelle de Jerzy - mais son évocation fréquente le transforme en un topos du portrait idéologique des Polonais goyim. Selon Audrey Kichelewski, l'antisémitisme est évoqué « dans la plupart des témoignages de ceux qui ont par la suite émigré de Pologne » ce qui constitue un « signe de sa forte prégnance dans la mémoire collective ${ }^{84} \gg$. Même s'il n'est pas présenté dans les œuvres du corpus comme l'apanage des seuls Polonais ${ }^{85}$, ces œuvres confirment les conclusions des analyses historiques synthétisées par Kichelewski.

L'antisémitisme des Polonais goyim fait donc indéniablement partie de la topique des souvenirs familiaux des Juifs d'origine polonaise émigrés en Belgique. Il s'agit non seulement d'un topos, mais aussi d'un véritable stéréotype discursif, c'est-à-dire un « lieu commun », répété d'une œuvre à l'autre ${ }^{86}$. Anne Herschberg-Pierrot a défini le stéréotype comme "une structure thématique [...] qui intègre un ou plusieurs prédicats obligés, ou constantes de prédicats ${ }^{87} »$. Pour les Polonais goyim, l'antisémitisme est justement un prédicat ou attribut quasi-obligé. Sa récurrence témoigne du fait qu'il s'agit bien d'une " image collective », autre définition du stéréotype ${ }^{88}$. L'existence du stéréotype de l'antisémitisme polonais est d'ailleurs attestée bien au-delà des œuvres de notre corpus, y compris dans d'autres pays, comme la France ou les États-Unis ${ }^{89}$. C'est le caractère répétitif, mais également généralisant, réductionniste et péjoratif (fortement chargé émotionnellement et axiologiquement) du portrait des Polonais goyim qui m'amène à parler de stéréotype ${ }^{90}$. Le réductionnisme est parfois poussé si loin que quasiment tout comportement des Polonais goyim peut se trouver ramené à de l'antisémitisme. Ainsi, pour donner un exemple extrême, le père d'Alain Berenboom va-t-il

82 Ibid.: 39.

83 Bar 2007: 82-83.

${ }^{84}$ Kichelewski 2018a: 48.

${ }^{85}$ Les personnages en font aussi l'expérience en Belgique ou en France. Ainsi, dans le texte de Hildebrand, Bassia (sic), une autre grand-tante maternelle de Jerzy, épouse un Français qui l'oblige à se convertir au catholicisme et à cacher ses origines juives, y compris à ses propres enfants. Son mari accepte d'accueillir Wanda chez eux seulement à condition qu'elle soit présentée comme Polonaise et catholique. Le propre fils de Bassia ne cesse de tenir des propos racistes. Dans L'apprentissage inutile, le héros-narrateur est terriblement humilié lorsque, sous l'occupation allemande, il se rend à l'Office du Travail et se déclare «Belge », en réponse à quoi le fonctionnaire flamand qu'il a devant lui le traite de « sale Juif » et lui crache à la figure. Dans J'avais 16 ans à Auschwitz, Henri Sonnenbluck raconte les rafles coorganisées à Anvers par des collaborateurs belges des nazis. Dans la nouvelle Eva du $20^{e}$ convoi du recueil de Zalek Kalb Beller, 1'hérö̈ne éponyme, après s'être enfuie d'un convoi à destination d'Auschwitz, est harcelée et violée par le boucher gantois qui la cache. Toutefois, ces comportements ne donnent pas lieu à des généralisations concernant tous les Belges (ou Français, dans le premier exemple).

${ }^{86}$ Certains auteurs signalent par ailleurs les liens entre stéréotype et topos en tant que « lieu commun » (voir Amossy 1991: 31).

${ }^{87}$ Cité d'après Amossy 1991: 31.

88 Voir ibid.: 10.

89 Voir Kichelewski 2018b: 479-480; Potel 2009: 33.

90 Voir Piontek 1993. 
jusqu'à prétendre que « les Polonais [...] dressaient [leurs chiens] à mordre les Juifs ${ }^{91} »$. La plupart des définitions insistent sur le lien indirect du stéréotype avec l'expérience et tel est effectivement le cas dans les œuvres du corpus : ce sont plutôt les ancêtres des auteur(e)s qui ont fait l'expérience de l'antisémitisme en Pologne et leur ont raconté leur vécu. L'image des Polonais goyim remplit donc la plupart des critères définitoires du stéréotype. En revanche, il ne s'agit pas, dans ce cas, d'une image sans lien avec la réalité (autre trait que certaines définitions attribuent au stéréotype) car, comme nous l'avons vu, des études historiques font bien état de la diffusion de l'idéologie antisémite dans de larges couches de la population polonaise au $\mathrm{XX}^{\mathrm{e}}$ siècle. Au-delà des origines référentielles indéniables de ce stéréotype, l'on peut s'interroger sur sa fonctionnalité. Shoshana Ronen a souligné le rôle structurant d'une stéréotypie discursive comparable pour la représentation de la Pologne chez les écrivains israéliens ${ }^{92}$. Le stéréotype de l'antisémitisme des Polonais goyim semble remplir une fonction similaire dans les œuvres de notre corpus. Néanmoins, il contribue moins à structurer les textes que la mémoire familiale, notamment en expliquant les raisons de l'émigration, et dote ainsi les trajets biographiques des immigré(e)s d'une certaine cohérence.

\section{Une problématisation difficile du stéréotype de l'antisémitisme polonais}

L'un des rares auteurs à problématiser explicitement le caractère stéréotypé de cette représentation est Alain Berenboom qui qualifie les opinions de ses parents sur les Po-

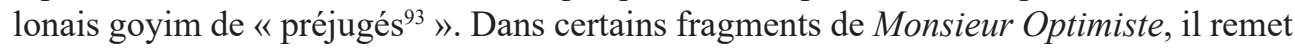
en question le stéréotype précité, mais d'autres passages illustrent la difficulté de s'en départir. Cet exemple montre que la conscience du stéréotype n'empêche pas nécessairement sa perpétuation. En parlant du vécu de sa grand-mère, Frania, qui a réussi à fuir le ghetto de Varsovie et à se cacher du côté « aryen », en se faisant embaucher par des paysans polonais, il suppose que « S'ils s'étaient doutés qu'elle était juive, elle aurait couru le risque d'être chassée, peut-être dénoncée aux Allemands ». Après quoi, il commente ses propres remarques d'une manière critique :

Vous êtes choqués ? [...] je me permets [...] de réduire les Polonais à une bande d'assassins antisémites, sans un mot sur leurs propres souffrances, le prix qu'ils ont eux aussi payé à la barbarie nazie, le courage dont la plupart firent preuve pendant la guerre et après, sous le joug soviétique. Ai-je le droit de décider en deux coups de cuiller à pot qui sont les bons et qui sont les mauvais avec aussi peu de nuances que mon père et ma mère ? Oui, c'est ainsi qu'ils parlaient des Russes et des Polonais (et des Ukrainiens et des Lituaniens). Ils ont l'excuse d'avoir vécu dans leur chair [...] la haine des Allemands, des Polonais et des Russes. Mais moi ? Aucun Allemand ne m'a jamais insulté, ni un Russe, ni un Polonais [...]. Et le seul qui m'ait traité de 'sale Juif !' était un brave Belge [...], issu de ce bon peuple tant vénéré par mes parents. Alors, de quel droit juger ces peuples - eux aussi ravagés par la tourmente - avec aussi peu de nuances $?^{94}$

\footnotetext{
91 Berenboom 2013: 153.

92 Ronen 2007: 301.

93 Berenboom 2013: 87.

94 Ibid.: 83-84.
} 
Berenboom présente souvent les Polonais goyim d'une manière réductionniste, faisant appel aux stéréotypes de l'ivrognerie ou de l'antisémitisme. Il semble lui-même gêné par le côté univoque de ce portrait. Il a conscience que ses propos relèvent de la stéréotypie, il en souligne le caractère réductionniste et généralisateur. Il met également en cause l'aspect médiatisé de son image des Polonais goyim, en se posant des questions sur son droit à juger négativement des gens qui ne lui ont jamais fait de mal car sa propre expérience de l'antisémitisme est liée à sa vie en Belgique. Écrivain confirmé, Berenboom semble partager cette « obsession du stéréotype » qui caractérise la littérature moderne ${ }^{95}$ et ressent le besoin de se justifier d'avoir recours à des lieux communs. Dans ce passage, il attribue la responsabilité de cette image stéréotypée à ses parents qui la lui auraient transmise. La seule justification qu'il trouve, c'est donc que son discours relève de la citation dans la mesure où il ne fait que rapporter l'opinion de ceux qui ont fait l'expérience de l'antisémitisme en Pologne. Mais cet argumentaire lui-même met encore une fois en exergue le caractère stéréotypé de sa représentation des Polonais goyim : n'ayant pas de contact direct avec eux, il répète des paroles d'autrui, participant à la diffusion d'une image de seconde main, autre définition possible du stéréotype. Finalement, l'auteur met explicitement en doute les paroles de ses parents, en supposant que «La vérité sur ce qu'ils pensaient des Polonais était sans doute plus nuancée que leurs discours ${ }^{96}$ ». Il se demande si « le jugement caricatural » de son père « à l'égard de ses compatriotes ${ }^{97}$ » ne relevait pas d'une stratégie d'adaptation, consistant à couper tous les ponts avec son pays d'origine, afin de mieux s'intégrer en Belgique. C'est pour cette raison que ses parents auraient « raillé l'antisémitisme de leurs compatriotes, sans leur reconnaître la moindre circonstance atténuante ${ }^{98} »$. Cette analyse paraît assez plausible dans la mesure où l'auteur rapporte les efforts de ses géniteurs pour devenir à tout prix les plus belges possible, y compris leur changement de nom («Berenbaum » devenant « Berenboom » qui sonne plus flamand). L'image réductionniste des Polonais goyim comme antisémites invétérés pourrait ainsi apparaître en tant que l'un des outils de construction d'une nouvelle identité belge des immigré(e)s.

Pourtant, cette analyse lucide des mécanismes psychologiques pouvant expliquer le caractère unidimensionnel de l'image que ses parents lui ont transmise n'empêche pas l'auteur de ressasser des lieux communs dans la suite de son livre. Lorsqu'il parle, juste après, des pogroms dont furent victimes certains Juifs revenant des camps, il invoque notamment les stéréotypes de la religiosité et de l'alcoolisme polonais :

[...] des Polonais, à peine libérés de la terreur allemande, s'étaient mis à massacrer quelques Juifs survivants venus se réinstaller dans leurs anciens villages, les tenant pour responsables de la férocité nazie, de la cruauté de Dieu et de la difficulté à dénicher une vodka convenable. Juste retour des choses : débarrassés des Juifs, ils héritèrent des communistes, ce qui améliora sensiblement la qualité de la vodka, mais pas leur sort ${ }^{99}$.

\footnotetext{
95 Voir Amossy 1991: 11-12.

96 Berenboom 2013: 84.

97 Ibid.: 87.

98 Ibid.

99 Ibid.
} 
Suggérant tout d'abord une explication des pogroms d'après-guerre liée au mécanisme du bouc émissaire, l'auteur retombe finalement dans la stéréotypie. L'antisémitisme des Polonais serait ainsi en partie dû à leur alcoolisme. Deux stéréotypes se rencontrent et l'un sert d'explication à l'autre. Ce fragment et d'autres qui apparaissent plus loin forment un contraste avec l'attitude compréhensive que prônait l'auteur dans le passage précité. Bien qu'il soit explicitement remis en doute, le stéréotype persiste. Cette incohérence me semble due à plusieurs facteurs. Premièrement, le caractère péjoratif de la plupart des stéréotypes invoqués permet de condamner les auteurs des violences, il joue donc un rôle éthique et argumentatif. Deuxièmement, ces lieux communs remplissent une fonction explicative. La compréhension de ces événements difficilement concevables de notre point de vue que constituent les pogroms d'après-guerre aurait exigé une analyse approfondie du contexte de l'époque, celui d'une anomie généralisée dans une société qui sortait de cinq ans de massacres de masse et de propagande antisémite (sans parler de siècles d'antijudaïsme propagé par l'Église catholique). Le stéréotype offre une compréhension immédiate et qui va de soi pour tous ceux et celles qui le partagent. En l'occurrence, il introduit aussi dans ce contexte grave une pointe d'humour (noir) dans la mesure où des mobiles risiblement triviaux sont attribués aux antisémites.

Dans le livre de Berenboom, l'on peut donc observer un conflit entre une vision stéréotypée des Polonais goyim et sa remise en question, et c'est cette première qui en sort victorieuse. Se confirme ainsi le caractère figé, "solidifié » du stéréotype, image difficilement modifiable, en accord avec l'étymologie du mot, «stereos» signifiant « solide» en grec $^{100}$. La « (mauvaise) conscience [...] du lieu commun ${ }^{101} »$ n'empêche pas Berenboom de le diffuser. L'auteur n'arrive pas à accepter une image de la patrie de son père contredisant le stéréotype, celle qui émerge des lettres que leurs proches restés au pays ont écrites en polonais et en yiddish et qu'il retrouve dans les papiers de sa mère pour se les faire traduire en français. Leur tonalité contraste sensiblement avec la vision de la Pologne héritée par l'auteur de ses parents. Tout d'abord, Berenboom présente ces lettres et cartes postales de ses grands-parents et de sa tante tels des « témoignages frais et bruts que l'écoulement du temps ou la mémoire n'avaient pas déformés ${ }^{102} »$. Ensuite, il cite des fragments de ces missives, et notamment de celles de son grand-père où l'on peut lire p.ex. : « [...] le calme règne non seulement chez nous à Maków, mais en général dans le pays, l'atmosphère est paisible ", pour tout de suite mettre en doute cette vision et lui opposer la sienne :

Que raconte Aba ? Ne voit-il pas ce qui se passe autour de lui ? La situation des Juifs est menacée par la terrible pression nazie aux frontières et, à l'intérieur de sa patrie, par un antisémitisme virulent qui se répand au même rythme fiévreux qu'en Germanie voisine. Des pogroms ensanglantent Brisk ${ }^{103}$ et Czestochowa, des partis nationalistes et des militaristes fascisants entrent dans le gouvernement ${ }^{104}$.

\footnotetext{
100 Amossy 1991: 13.

101 Ibid:: 193.

102 Berenboom 2013: 112.

103 Version yiddish du nom de la ville de Brześć.

104 Berenboom 2013: 116.
} 
Berenboom semble incapable d'accepter que son grand-père ait pu vivre en Pologne une vie paisible peu avant le déclenchement de la Seconde Guerre mondiale. Il juge son témoignage à la lumière du savoir historique concernant la fascisation du régime polonais de l'époque, la croissance de l'antisémitisme et la Shoah toute proche, ainsi que de l'image des Polonais goyim qui lui a été transmise par ses parents. Il barre d'un trait de plume une vision plus positive, la considérant comme preuve de l'aveuglement de son grand-père. Pour l'auteur, « la douceur [...] du shtetl polonais » ne peut être que « faussement tranquille ${ }^{105} »$. Un savoir a posteriori et le stéréotype priment sur un témoignage d'époque.

La tension entre le stéréotype et sa remise en cause est constante dans le texte de Berenboom. D'une part, l'auteur reconnaît explicitement que la Lituanie et la Pologne sont « deux taches blanches sur la carte de [s]a mémoire ${ }^{106} »$. Il souligne que, en raison de l'éloignement et des destructions dues à la guerre, même pour son père, qui y avait vécu, Varsovie est devenue «Une ville imaginaire, à jamais disparue » et « une cité de conte de fées, irréelle et magique ${ }^{107} \gg$. Il ne cesse donc d'insister sur le caractère imaginaire de sa représentation de la Pologne et la distance qui la sépare du pays réel. D'autre part, toutefois, il continue à multiplier dans son texte des remarques catégoriques sur les caractéristiques de la vie juive en Pologne et en Europe centrale en général, présentée comme « vie tragique », « vie triste, dure, souvent sanglante », et ainsi décrite : «Des catastrophes épouvantables ? Des événements terribles ? C'est le lot quotidien de la vie des Juifs d'Europe centrale. Massacres, pogroms, maladies, expulsions ${ }^{108} »$. Ce ne sont pas uniquement les Polonais goyim qui sont stéréotypisés, mais également la Pologne juive. Son image est non seulement misérabiliste, mais aussi catastrophique, et contraste fortement avec celle qui se dégage non seulement des lettres du grand-père de l'auteur, mais aussi des travaux historiques sur l'extraordinaire foisonnement de la vie culturelle, intellectuelle et religieuse de la communauté juive en Pologne avant la Shoah. Des livres d'histoire, Berenboom ne retient que ce qui concorde avec la vision héritée de ses parents, et notamment l'insistance sur la violence des conflits entre Polonais juifs et Polonais goyim. C'est une opinion préconçue qui détermine son utilisation des sources historiques. Dans une sorte d'orientalisme intra-européen, une Pologne misérable s'oppose à une Belgique présentée comme «le vrai monde ${ }^{109} »$, celui que son père a toujours rêvé de rejoindre. D'autres auteur(e)s abondent dans le même sens. Dans Plus que la vie, le principal souvenir que Charles Lerner garde du voyage effectué avec sa mère en Pologne, est celui de son « ahurissement de découvrir des enfants pieds nus dans le stetl (sic) $)^{110} »$. Nathalie Skowronek confirme, dans Un monde sur mesure, le caractère mythique de l'Occident pour les Juifs est-européens, en disant que « la jeunesse juive » était «attirée par la mythologie de $l^{\prime}$ Ouest ${ }^{111}$ ». Cette Occident mythique incarne les plus hautes valeurs. Yitzhak, l'un des personnages de la pièce d'Anna Stelkowicz, affirme : « Pour nous, Juifs de Pologne, les pays de l'Europe occidentale incarnaient les

\footnotetext{
105 Ibid.: 136.

106 Ibid.: 227.

107 Ibid.: 181.

108 Ibid., respectivement: 166, 164.

109 Ibid.: 105.

110 Lerner 2007: 28-29.

111 Skowronek 2017: 30.
} 
rendez-vous avec la liberté112 ${ }^{\prime \prime}$ ce qui sous-entend la vision de l'Est comme une sorte de prison de laquelle il fallait s'échapper. Julek Bar intitule justement le chapitre racontant son arrivée en Belgique «La liberté ». L'on voit ainsi qu'une forme d'orientalisme, compris, à la suite d'Edward Said, comme idéologie instaurant un rapport hiérarchique entre un Occident survalorisé et un Orient dévalorisé, existe aussi dans la perception des pays de l'Europe de l'Est par les émigrés juifs et leurs descendants. Alors qu'au Moyen Âge, c'est vers cette région que se sont dirigés les Juifs fuyant les persécutions en Europe occidentale, vers la fin du XIX ${ }^{\mathrm{e}}$ siècle et au début du XX', le mouvement s'inverse. L'insistance sur la misère et l'antisémitisme s'inscrivent dans une idéologie orientaliste qui accompagne ce changement.

\section{L'étrangéisation linguistique et son dépassement possible}

Dans le portrait des Polonais goyim, à côté de leur antisémitisme, qui les met à distance des sujets parlants, c'est aussi leur langue qui joue le rôle d'un facteur d' « étrangéisation ». Cette dernière touche aussi parfois les Juifs polonais émigrés eux-mêmes, comme on le voit dans cette affirmation du héros-narrateur de L'apprentissage inutile de David Scheinert : «C'était la Pologne qui m'avait d'abord fait différent de ceux qui m'entouraient. Elle m'avait donné une autre langue que celle de la Belgique, un accent bizarre $^{113}$ ». La langue apparaît tel un concentré de «polonité » et d'étrangeté. Un Polonais, y compris lorsqu'il est juif, est parfois rendu étrange(r) non seulement par sa langue maternelle, mais aussi son usage fautif du français. Dans une lunetterie où il travaille comme apprenti pendant un certain temps, le héros-narrateur du livre de Scheinert rencontre un autre Juif polonais dont il cite notamment ces deux répliques : « Vous compris? » et « Alors, hein, quoi vous dis ? ${ }^{114} \gg$. Les fautes linguistiques emblématisent le statut du personnage, celui d'étranger. Quand le protagoniste du roman de Scheinert enseigne le français à des réfugiés juifs allemands, autrichiens et polonais, il utilise leur maladresse linguistique pour les ridiculiser. Une Polonaise refuse de se laisser faire et lui déclare : «Pardonnè-moua, mais jè nè sais pas faire rire... ${ }^{115}$ ». Les particularités de sa prononciation sont rendues par une orthographe modifiée, mais la réplique qu'elle prononce signifie sa révolte contre une étrangéisation linguistique humiliante. Cette dernière pourrait paraitre une forme « douce » de xénophobie mais au moment de l'occupation allemande, lors des rafles, comme le constate le protagoniste lui-même, «l'accent slave » est l'un des " pièges sûrs ${ }^{116}$ ». À côté des critères raciaux, au demeurant peu sûrs, l'accent polonais peut devenir un critère discriminant décisif et mener un immigré juif dans un camp de concentration. C'est le versant tragique de l'étrangéisation linguistique. Cette dernière touche aussi bien les Polonais goyim que certains Juifs polonais.

\footnotetext{
112 Stelkowicz 1997: 8.

113 Scheinert 1985: 32.

114 Ibid.: 113.

115 Ibid:: 138 .

116 Ibid.: 339.
} 
Dans Le livre de Joseph de Bernard Dan, le héros-narrateur place justement l'histoire de son séjour à Varsovie sous le signe de l'étrangeté linguistique, s'exclamant dès le début du récit : «Quelle langue bizarre !117 ». Et cette bizarrerie le fatigue : «Ces cédilles, ces accents et ces biffures m'étourdissent. Ça me rappelle ces exercices pénibles que Maman-Régine m'imposait sur prescription de l'ophtalmologue... ${ }^{118} \gg-$ avoue-t-il encore à la vue d'un texte polonais. Il commente le nom de famille de l'un des participants du congrès de dentistes auquel il participe - « Szwierszcz » (déformation probable de « Świerszcz ») - de la manière suivante : « Ce conglomérat de consonnes à la marge d'un unique couple de voyelles [...] m'a fasciné. J'ai eu l'impression de découvrir la Pologne ${ }^{119}$ ». Nous avons ici affaire à un exotisme linguistique : les particularités orthographiques et phonétiques du polonais deviennent objet de fascination et métonymie du pays de ses locuteurs. Mais le personnage ne s'en tient pas à l'exotisation. Il essaie d'apprivoiser l'étrangeté linguistique en la ramenant à du connu par des transcriptions phonétiques « francisantes » p.ex. de noms propres polonais. De cette manière, le nom de famille cité ci-dessus se trouve transformé en " chchviirchtchch ${ }^{120}$ ». L'auteure de La danseuse sur un nuage orthographie à la française des expressions ou phrases polonaises entières, p.ex. « Dayimispokoui » ou « nazdroviè ${ }^{121}$ ». Cette pratique va au-delà de l'orientalisme linguistique évoqué au début de cette étude à propos de l'orthographe fautive et de la francisation de certains mots : elle témoigne d'une connaissance rudimentaire (surtout orale) du polonais et peut-être même d'une volonté d'en transmettre des notions aux lecteurs et lectrices francophones. En effet, cette orthographe francisée leur permettra de prononcer à peu près correctement les expressions et phrases citées.

La citation de vocables polonais peut aussi servir à des jeux de mots à valeur identitaire. Le protagoniste du Livre de Joseph interprète le nom de la compagnie aérienne polonaise LOT à la lumière de la signification du mot français « lot » et d'une référence intertextuelle biblique :

Ils ne manquent pas d'humour - noir. Lot comme le destin, comme la loterie. Je suis perdant. Comme la destruction de Sodome. Et de Varsovie. Et sa femme taraudée par la curiosité, qui se retourne pour contempler les ruines. Elle est punie : pétrifiée sur place. Elle n'ira pas plus loin. Et moi ? ${ }^{122}$

Un nom propre polonais, bien adapté à désigner une compagnie aérienne, puisqu'il signifie « vol», se trouve confondu à la fois avec son homographe français et le nom d'un personnage biblique. Le héros-narrateur du roman de Dan, scrutant le passé de sa famille, se compare à la femme de Lot, punie pour s'être retournée afin de regarder une dernière fois la ville de Sodome. Et il s'interroge sur l'issue de sa quête des origines, suggérant par la référence biblique que ce retour sur le passé familial pourrait s'avérer dangereux. Immergé dans un univers linguistique qui lui est étranger, Jean-Paul Rakover tente de se l'approprier en transposant un mot dans sa langue maternelle et en le reliant

117 Dan 2014: 8 .

118 Ibid.: 53.

119 Ibid.: 49.

120 Ibid.

121 Cykiert 2013: 79-80.

122 Dan 2014: 47. 
à sa situation. Cet élément d'une langue tout d'abord exotisée et présentée comme non seulement étrangère mais aussi étrange se trouve de la sorte ramené à quelque chose de familier et inscrit dans la quête intime du personnage. Grâce à un début d'appropriation dont il fait l'objet, le polonais n'est plus seulement un facteur d'étrangéisation, il devient instrument d'une quête identitaire. À la recherche de l'identité de ses ancêtres, le héros joue avec les mots, et le détournement francisant auquel il les soumet peut symboliser sa position intermédiaire : Français par sa citoyenneté, sa langue et sa culture, il est aussi Juif polonais par ses origines. En francisant des vocables polonais, le protagoniste peut se réapproprier dans une certaine mesure ce qui lui apparaissait initialement comme étrange et étranger. Une certaine forme de «polonité linguistique» s'avère en partie assimilable. La distance par rapport à la Pologne, mise en place chez d'autres auteur(e)s par le recours au stéréotype, est ici dans une certaine mesure abolie par la création d'une interlangue franco-polonaise.

\section{Conclusion}

Au moment de découvrir ses origines polonaises, le narrateur premier du Livre de Joseph de Bernard Dan commence par énumérer tout ce qu'il associe à la Pologne :

Que m'évoque la Pologne ? Rien. Le mot ubuesque d'Alfred Jarry cité hors contexte en classe d'histoire : en Pologne, c'est-à-dire nulle part. [...] La vodka. Saoul comme toute la Pologne. Solidarnosc. Travailler comme un Polonais : dur, bien, pas cher. Un plombier polonais. Copernic. Marie Curie. Et bien sûr, mon homonyme ${ }^{123}$.

Cette énumération commence par le constat d'un vide : «rien », et se poursuit par l'évocation d'un non-lieu : « nulle part », emprunté à un discours d'Alfred Jarry accompagnant la première représentation d'Ubu roi où l'auteur déclarait : "l'action [...] se passe en Pologne, c'est-à-dire Nulle Part ${ }^{124} »$. Malgré ces deux négations, suit immédiatement une série d'éléments hétérogènes associés à la Pologne ; à côté d'une boisson emblématique, y figurent trois expressions, dont deux - « saoul comme toute la Pologne », variante de « saoul comme un Polonais », et «plombier polonais », exprimant la crainte d'une concurrence déloyale de la part de travailleurs polonais au moment de l'adhésion de la Pologne à l'Union européenne - ont une résonance négative, la troisième - « travailler comme un Polonais »- beaucoup plus positive. Toutes ces associations renvoient à des stéréotypes. S'y ajoutent des références à des événements ou personnages historiques. Dans cette évocation de la Pologne, l'on passe du néant (« rien », « nulle part ») à une liste hétéroclite. Dans l'esprit du personnage, l'image du pays se compose d'une collection de bribes discursives disparates, en majorité stéréotypées. Elle oscille entre l'inexistence et le tohu-bohu qui témoigne de l'absence d'une représentation cohérente du pays. Le rôle primordial qu'y joue la stéréotypisation et l'ambivalence qui se dégage des références convoquées font que l'on peut considérer ce fragment comme exemplaire

${ }^{123}$ Ibid.: 16-17. L'homonyme en question, c'est le pape Jean-Paul II dont le héros partage le prénom composé.

124 Jarry 1972a: 401. 
de la façon dont la Pologne et les Polonais goyim sont représentés dans la plupart des œuvres du corpus.

Les critiques et historiens qui se sont jusqu'à présent penchés sur la perception de la Pologne par les émigrés juifs, y compris les écrivains, dans différents pays, insistaient le plus souvent sur la coloration émotionnelle négative, ou pour le moins ambiguë, de leur rapport à leur pays d'origine. Kamila Dąbrowska parle d' « images [...] teintées d'amertume $^{125} »$, Karolina Famulska-Ciesielska fait état d'un « ressentiment à l'égard de la Po$\operatorname{logne}{ }^{126}$ » et Pascale Falek constate que « La relation des Juifs polonais à leur pays d'origine est généralement perçue comme tragique et amère ${ }^{127}$ ». Même si les trois auteures citées remarquent aussi dans cette relation une ambiguiité, la représentation de la Pologne étant empreinte d'une certaine nostalgie, la négativité semble quand même dominante. Ces images littéraires paraissent s'inscrire dans une tradition mémorielle plus large, issue principalement de l'expérience de la Shoah que Havi Ben-Sasson résume ainsi :

La montée graduelle en eux d'un sentiment d'amère déception parce qu'ils constataient de plus en plus l'indifférence, voire la malveillance de la population polonaise pendant les déportations et l'extermination des Juifs, a donné lieu à un sentiment de grande hostilité à l'encontre des Polonais, sentiment qui a ensuite dominé la mémoire collective d'après la guerre et une grande partie de l'historiographie juive ${ }^{128}$.

Les pogroms d'après-guerre et la campagne antisémite orchestrée par le parti communiste polonais en 1968 ont sans doute noirci encore davantage les souvenirs des émigrés juifs. La plupart des œuvres de notre corpus ne remettent pas vraiment en cause cette coloration de la mémoire collective des Juifs d'origine polonaise ; les portraits des Polonais goyim y paraissent dotés d'une forte charge émotive, parfois ambivalente, mais le plus souvent négative, y compris lorsqu'ils concernent d'autres époques que celle de la Seconde Guerre mondiale. L'attitude de Lili, l'arrière-grand-mère paternelle de Nathalie Skowronek, qui a quitté la Pologne dans les années 1920, et qui affirme catégoriquement : « Retourner chez les Polaks ? Ça jamais ${ }^{129}$ ", pourrait ainsi être emblématique du rapport de nombreux immigrés juifs d'origine polonaise qui désignent également souvent leurs anciens concitoyens par des termes aux connotations péjoratives, confirmant la force de leur rancœur.

Cependant, la Pologne et les Polonais goyim sont le plus souvent mentionnés dans leurs œuvres seulement « en passant ». Il ne s'agit pas ici de textes qui leur seraient principalement consacrés et qui en proposeraient des portraits « en pied ». Le pays d'origine des immigré(e)s et ses habitants non juifs sont évoqués surtout lors de la présentation des raisons de l'émigration et des souvenirs liés à l'époque passée en Pologne. Ils sont mentionnés de manière plutôt circonstancielle. Leur présence dans les textes analysés prend des formes éphémères. Le caractère stéréotypé des images tient peut-être aussi à leurs dimensions réduites : brossées en quelques traits de plume rapides, elles ne sauraient déboucher sur une vision multidimensionnelle et approfondie. Quant aux contenus de la

\footnotetext{
125 Dąbrowska 2011.

126 Famulska-Ciesielska 2011.

127 Falek 2011.

128 Ben-Sasson 2009: 122.

129 Skowronek 2017: 30.
} 
représentation, bien plus que l'image d'un nulle part qu'évoque la formule trop célèbre et souvent mal interprétée, car citée dans une version tronquée, d'Alfred Jarry ${ }^{130}$, c'est celle d'un ailleurs que la Pologne semble inspirer aux immigrés juifs d'origine polonaise et à leurs descendants. Un ailleurs irrémédiablement « autre » et étranger, souvent hostile. Également, un immense lieu de mémoire, renvoyant non seulement à la catastrophe de la Shoah, mais, plus largement, au passé de nombreuses familles juives. En général, les auteur(e)s évoquent moins souvent l'espace géographique polonais en soi que le « pays vécu » ou remémoré, ainsi que les relations, parfois difficiles, avec ses habitants goyim. Ces derniers font souvent l'objet d'un portrait à charge.

L'examen du corpus composé de textes d'immigré(e)s d'origine juive polonaise en Belgique francophone et de leurs descendants permet ainsi, sinon de contredire, en tout cas de nuancer sérieusement les conclusions de l'article pionnier d'Agnieszka Pantkowska sur l'image de la Pologne et des Polonais dans les lettres belges de langue française. L'auteure concluait à une image positive, liée notamment à des facteurs historiques (p.ex. l'interdépendance entre l'insurrection de novembre polonaise et l'indépendance belge). Elle parlait même d' « une image très positive de la Pologne et des Polonais ${ }^{131}$ » chez les écrivains belges francophones. L'analyse de notre corpus, prenant en compte des textes que la chercheuse a écartés du sien ${ }^{132}$, ne confirme pas cette généralisation. Au contraire, l'image qui s'en dégage est beaucoup plus ambiguë et penche plutôt du côté négatif, même si les éléments positifs n'en sont pas entièrement absents (p.ex. dans les évocations de la nature, de la vie familiale ou des traditions de la communauté juive polonaise). Cette représentation de la Pologne, mais surtout des Polonais, se distingue clairement de celle qui semble avoir prévalu pendant longtemps en Belgique. Si le pays luimême est encore assez souvent représenté comme locus amoenus, ses habitants non juifs devraient plutôt être désignés populus horribilis, malgré quelques portraits individuels plus positifs. Quant aux racines de cette image, au-delà d'un antisémitisme bien réel dont la diffusion en Pologne à certaines époques, notamment dans l'entre-deux-guerres, est historiquement attestée ${ }^{133}$ et dont le souvenir reste très présent dans les familles juives, il pourrait s'agir, comme le suggère l'exemple des parents d'Alain Berenboom analysé ci-dessus, d'une stratégie identitaire adoptée par de nombreux immigrés qui font tout pour se distancier de leur pays d'origine, vraisemblablement afin de mieux s'identifier à leur pays d'accueil. Ce besoin de «distanciation identitaire » a probablement contribué à négativiser l'image de la Pologne chez les immigré(e)s car cette dernière semble plus nuancée et multidimensionnelle chez les Juifs qui y sont restés ${ }^{134}$. Si l'étude de la

130 Voici la suite de son raisonnement : « Nulle Part est partout, et le pays où l'on se trouve, d'abord » (Jarry 1972b, p. 402). Par sa formule, Jarry visait donc une universalisation du sens de la pièce et non sa localisation en Pologne.

131 Pantkowska 2006: 129.

132 Elle déclare explicitement : « [...] nous n'avons pris en considération que les écrivains belges qui n'ont pas d'origines polonaises afin de mieux observer le fonctionnement du stéréotype » (Pantkowska 2006 : 122), sans vraiment expliquer pourquoi un tel rétrécissement du corpus permettrait de « mieux observer» ce fonctionnement. Les Belges d'origine polonaise seraient-ils « moins belges » que ceux « de souche »?

133 En faisant référence notamment à des recherches historiques (Jan T. Gross), littéraires (Maria Janion) et anthropologiques (Joanna Tokarska-Bakir), Audrey Kichelewski va jusqu'à parler de l'antisémitisme en Pologne comme « vision constitutive du monde » et « code culturel largement partagé » (Kichelewski 2009: 1104).

134 Kichelewski 2018b: 483-484. 
représentation de la Pologne et des Polonais goyim chez les immigrés juifs d'origine polonaise devait être poursuivie à propos d'autres pays d'accueil, il me semble qu'elle devrait justement prendre largement en compte les enjeux identitaires de cette représentation pour les immigrés eux-mêmes. Certes, ces derniers emmènent dans leurs bagages (mentaux) des souvenirs de leur pays natal, mais aussi bien eux que leurs descendants les retravaillent ensuite, une fois arrivés dans leur pays d'installation. Une contrée autrefois proche et familière peut s'y transformer en un ailleurs qui prend des allures de plus en plus inquiétantes, et la distance géographique est susceptible de se doubler d'une distanciation psychologique et émotionnelle croissante.

\section{BIBLIOGRAPHIE}

\section{Corpus}

Bar, J. (2007), Une part de notre humanité, Nivelles (éd. électronique).

Berenboom, A. (2013), Monsieur Optimiste, Bruxelles.

Cykiert, R.-S. (2013), La danseuse sur un nuage, Liège.

Dan, B. (2014 [2011]), Le livre de Joseph, La Tour d'Aigues.

Gutmacher, S. (2010), Auschwitz-Dachau. Novembre 1942-mai 1945. Témoignages, poèmes, contes, Paris.

Hildebrand, J. (2013), Wanda. De la Sibérie à Anvers, le courage de la différence, Bruxelles (éd. électronique).

Jarry, A. (1972a), Discours d'Alfred Jarry prononcé à la première représentation d'Ubu roi, in: idem, Euvres complètes. T. 1, Paris : 400-401.

Jarry, A. (1972b), Autre présentation d'Ubu roi, in : idem, Euvres complètes. T. 1, Paris : 401-403.

Kalb Beller, Z. (1978), L'immigré Herschel Schaerbeeker raconte, Paris-Bruxelles.

Kalisky, R. (1990), Aïda vaincue, Paris.

Kalisky, R. (2006 [1981]), Belgique? Le pays le plus imaginaire du monde, in : Quaghebeur, M., Anthologie de la littérature française de Belgique. Entre réel et surréel, Bruxelles : 296-301.

Kichka, H. (2005), Une adolescence perdue dans la nuit des camps, Bruxelles.

Kichka, M. (2012), Deuxième génération. Ce que je n'ai pas dit à mon père, Paris.

Lerner, Ch. (2007), Plus que la vie, Nivelles.

Nysenholc, A. (2007), Bubelè. L'enfant à l'ombre, Paris.

Scheinert, D. (1985 [1948]), L'apprentissage inutile, Bruxelles.

Skowronek, N. (2017), Un monde sur mesure, Paris.

Sonnenbluck, H. (1990), J'avais 16 ans à Auschwitz, Bruxelles.

Stelkowicz, A. (1997), Eva, Rachel, Yitzhak et les autres... De l'émigration polonaise à l'enfant caché, tapuscrit conservé aux Archives et Musée de la Littérature de Bruxelles, cote MLTB 02453.

Szlingerbaum, S. (1989), Bruxelles-transit. Bruxelles.

\section{Ouvrages critiques}

Amossy, R. (1991), Les idées reçues. Sémiologie du stéréotype, Paris.

Bakhtine, M. (2013), Esthétique et théorie du roman, trad. D. Olivier, Paris.

Ben-Sasson, H. (2009), La Pologne et les Polonais vus par les Juifs polonais pendant la Shoah : un regard qui a évolué, trad. M. Halpern, in : Szurek, J.-Ch., Wieviorka, A. (dir.), Juifs et Polonais. 1939-2008, Paris: 109-122.

Bordas, É., Barel-Moisan, C., Bonnet, G., Déruelle, A., Marcandier, Ch. (2015), L'analyse littéraire, Paris.

Cała, A., Węgrzynek, H., Zalewska, G. (2000), Historia i kultura Żydów polskich. Słownik, Varsovie. 
Casanova, P. (2002), Consécration et accumulation de capital littéraire. La traduction comme échange inégal, Actes de la recherche en sciences sociales 144: 7-20.

Dąbrowska, K. (2011), La signification changeante des souvenirs matériels chez les immigrés juifs polonais d'après-guerre en Israël, trad. J. Grumbach, Bulletin du Centre de recherche français à Jérusalem 22, https://journals.openedition.org/bcrfj/6555 (consulté le 7 juin 2019).

Falek, P. (2011), Une relation douce-amère. La Pologne et les étudiants juifs polonais en Belgique pendant l'entre-deux-guerres, Bulletin du Centre de recherche français à Jérusalem 22, https:// journals.openedition.org/bcrfj/6472 (consulté le 7 juin 2019).

Famulska-Ciesielska, K. (2011), Le souvenir de la Pologne dans la littérature polonaise en Israël, trad. M. Rey, Bulletin du Centre de recherche français à Jérusalem 22, https://journals.openedition.org/ bcrfj/6464 (consulté le 7 juin 2019).

Flem, L. (1989), Bruxelles-transit - Bruxelles-transfert, in: Szlingerbaum, S., Bruxelles-transit, Bruxelles: 93-97.

Głowacka, D. (2016), Współ-pamięć, pamięć „negatywna” i dylematy przekładu w „wycinkach” z Shoah Claude'a Lanzmanna, Teksty Drugie 6: 297-311.

Gross, J.T. (2014), Moisson d'or. Le pillage des biens juifs, trad. P.-E. Dauzat, Paris.

Kichelewski, A. (2009), La peur des Juifs ou des Juifs qui ont peur? Fear de Jan T. Gross et les débats sur l'antisémitisme en Pologne, Annales. Histoire, sciences sociales 5: 1091-1104.

Kichelewski, A. (2018a), Les survivants. Les Juifs de Pologne depuis la Shoah, Paris.

Kichelewski, A. (2018b), In or Out? Identities and Images of Poland among Polish Jews in the Postwar Years, in: Polonsky, A.; Węgrzynek, H. and Żbikowski, A. (dir.), New Directions in the History of the Jews in the Polish Lands, Boston: p. 472-484 (éd. électronique).

Libionka, D. (2009), L’État clandestin polonais et la 'question juive' (1942-1944), trad. M. Szymańska, in : Szurek, J.-Ch., Wieviorka, A. (dir.), Juifs et Polonais. 1939-2008, Paris: 61-77.

Pantkowska, A. (2006), Le stéréotype de la Pologne et des Polonais dans les lettres belges de langue française, in : Forycki, M., Serwański, M. (dir.), Amis et ennemis héréditaires. Les stéréotypes nationaux, Poznań: 117-129.

Piontek, D. (1993), Stereotyp. Geneza, cechy, funkcje, in: Borowczyk, K.; Pawełczyk, P. (dir.), W kregu mitów i stereotypów, Poznań-Toruń: 20-40.

Potel, J.-Y. (2009), La fin de l'innocence. La Pologne face à son passé juif, Paris.

Ronen, S. (2007), Polin. A Land of Forests and Rivers. Images of Poland and Poles in Contemporary Hebrew Literature in Israel, Varsovie.

Scherr, L. (1989), Bruxelles-transit et la tradition du film yiddish, in : Szlingerbaum, S., Bruxelles-transit, Bruxelles: 79-83.

Schreiber, J.-P. (2004), L'immigration juive en Belgique, du Moyen Âge à nos jours, in : Morelli A. (dir.), Histoire des étrangers et de l'immigration en Belgique, de la préhistoire à nos jours, Bruxelles: 215-242.

Tollet, D. (1992), Histoire des Juifs en Pologne du XVI siècle à nos jours, Paris. 